\title{
Long-term reliability of fiber-optic current sensors in damp heat
}

\author{
Miklós Lenner, Lin Yang, Andreas Frank, Klaus Bohnert \\ ABB Switzerland Ltd., Corporate Research Center, Segelhofstr. $1 \mathrm{~K}, \mathrm{CH}-5405$ Baden-Dättwil, \\ Switzerland \\ miklos.lenner@ch.abb.com
}

\begin{abstract}
The measurement of electric current in industrial applications is associated with stringent requirements for accuracy, reliability and availability. We set up an extensive reliability test program in order to validate the reliability of components used in fiber-optic current sensors (FOCS). For many components operated in harsh environments, damp heat is often the most crucial environmental stressor. Potential failure modes include shifts in the source wavelength, changes in birefringence of certain optical components due to stress and excessive insertion loss. Degradation can lead to sensor scale factor change, or in extreme cases, to sensor failure. Our experiments have demonstrated that appropriately designed, packaged and burned-in components survive extended exposure to damp heat without significant performance loss.
\end{abstract}

Key words: Fiber-optic current sensors, reliability, availability, harsh environments

\section{Introduction}

In the past decade, fiber-optic current sensor (FOCS) technology [1]-[5], gained remarkable attention in several industrial applications, in particular in the electro-winning of metals and high-voltage (HV) substation automation [6]-[11], (Fig. 1). The optical measurement of electric current enables accuracies up to $\pm 0.1 \%$, both for direct and alternating currents, in a large operating temperature range (e.g., -40 to $85^{\circ} \mathrm{C}$ ) and provides several advantages over the conventional Hall effect-based and inductive current transformers including substantial reduction of weight, size and footprint [10], no magnetic saturation at fault currents and digital communication. Fiber-optic current sensors measure the differential magneto-optic phase shift between left- and right circularly polarized light waves in a coil of fiber (Fig. 2) [4] that is linearly proportional to the electric current (Faraday effect). In order to retrieve the magneto-optic phase shift interferometric fiberoptic current sensors employ an optical phase modulator, which dynamically shifts the working point of the interferometer into the linear range of the sinusoidal response function of the sensor and, by using a closed-loop feedback circuit [12], compensates the differential magneto-optic phase shift of the light waves produced by the current. In addition to the technical requirements, HV applications require that specifications must

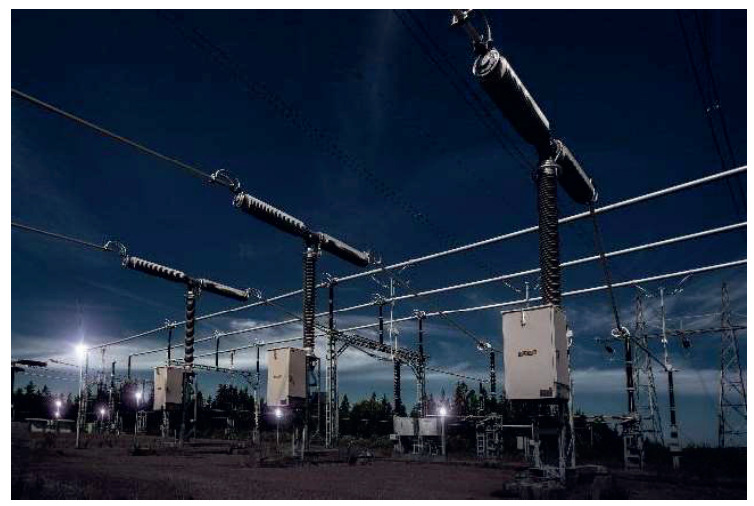

Fig. 1: High voltage circuit breakers in an electric power transmission substation with integrated fiberoptic current sensors [10].

be preserved over the entire lifetime of the equipment - that is typically over 30 years for conventional current transformers. Concerning the optics, this translates into preserving the source wavelength [13] and the polarization states of the light waves [14] within a fraction of $0.1 \%$ over extended periods of time. This sets stringent reliability requirements for the components used.

Thus, we performed a thorough reliability test program for the components, which included temperature cycling, constant high temperature and damp heat tests. For operation in harsh environments, damp heat is the most established accelerated testing method, which 


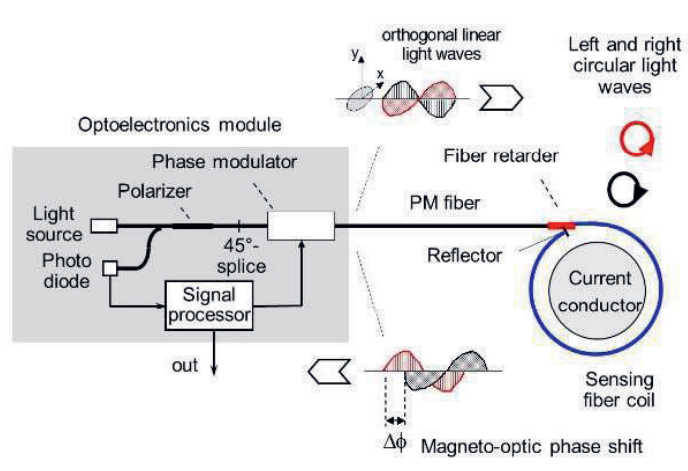

Fig. 2: Fiber-optic current sensor.

can cause severe impact over short testing time [15]. Testing at elevated temperature and humidity effectively accelerates chemical processes which lead to material ageing and also promotes penetration of humidity through the sealing of component packages.

In this paper, we focus on two of the most critical components of the sensor (the superluminescent diode source (SLED) and phase modulator) and show that they survive high relative humidity $(85 \%)$ at elevated temperature $\left(65^{\circ} \mathrm{C}\right)$ without significant performance degradation.

\section{Results}

Prior to ageing the characteristic parameters of the components were measured for reference. Subsequently, the components were subjected to damp heat for defined periods of time. The environmental conditions (temperature $T=65^{\circ} \mathrm{C}$, relative humidity $R h=85 \%$ ) were set according to [15] in a climatic chamber. As for the ageing temperature, we took the maximum operating temperature of the components into consideration in order to avoid the generation of "trivial" failures, i.e. failure modes that are associated with the operation of a device outside the specifications. After each ageing interval, the components were re-characterized and the results were compared with the initial reference data.

In fiber-optic current sensors, the proportionality between electric current $I$ and the magneto-optic phase shift $\Delta \phi$ is given by the Verdet constant $V$ of the fused silica sensing fiber. Due to the wavelength dispersion of the Verdet constant, roughly in proportion to $1 / 2 \lambda$ [16], reliable sensor operation requires that the central wavelength of the light source remains stable over the entire mission. For example, at $\lambda_{0}=1310 \mathrm{~nm}$ central wavelength, a variation of $\Delta \lambda= \pm 650 \mathrm{pm}$ would lead to a sensor scale factor variation of $\pm 0.1 \%$.

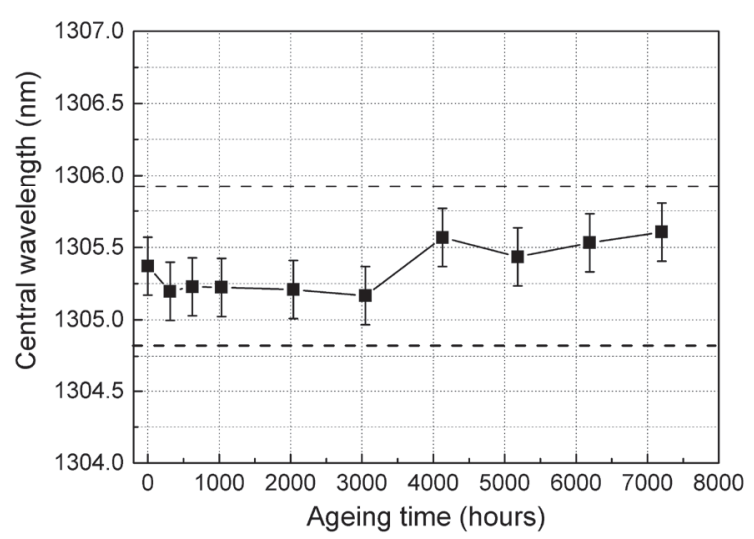

Fig. 3: SLED central wavelength vs ageing time at $65^{\circ} \mathrm{C}$ and $85 \%$ relative humidity. The dashed lines represent the wavelength shift corresponding to a scale factor shift of $\pm 0.1 \%$.

Fiber-optic current sensors typically use semiconductor light sources (e.g. SLEDs) that are generally characterized by high temperature dependence (typically in the order of $500 \mathrm{pm} /{ }^{\circ} \mathrm{C}$ ). Thus, it is essential that stable wavelength operation is ensured by a thermo-electric cooler (TEC) that is usually integrated in the device package. Ageing of the SLED itself may give rise to wavelength changes but the degradation of any of the thermal interfaces between the light emitting chip, the TEC and the environment may also affect the performance of the thermal control and thus the stability of the central wavelength.

While wavelength drift, if any, due to normal ageing is expected to take place gradually over time, moisture ingress in the temperaturecontrolled package may lead to sudden failures, e.g., due to condensation and consequent fast degradation of the optical surfaces (chip facet and fiber coupling) and materials (chip, adhesives, wire bonds, heat transfer) due to oxidation [17].

The reliability tests were performed on SLEDs from several manufacturers. The nominal central wavelength and the full width at half maximum (FWHM) of the optical spectrum were $\lambda_{0}=1310$ $\mathrm{nm}$ and $\approx 40 \mathrm{~nm}$, respectively. The optical output power was rated at $5 \mathrm{~mW}$. The chip temperature was set to $T_{\text {chip }}=25^{\circ} \mathrm{C}$, similarly to field conditions. The devices were mounted according to the sensor production specifications and tested in a climatic chamber including the electric connections. During ageing, the SLEDs were operated at $70 \%$ of the maximum rated driving current. Prior to each spectral measurement, the optical spectrum analyzer (OSA) was recalibrated. The results of a representative sample are depicted in Fig. 3 . The overall wavelength accuracy of the measurement system was estimated to be 


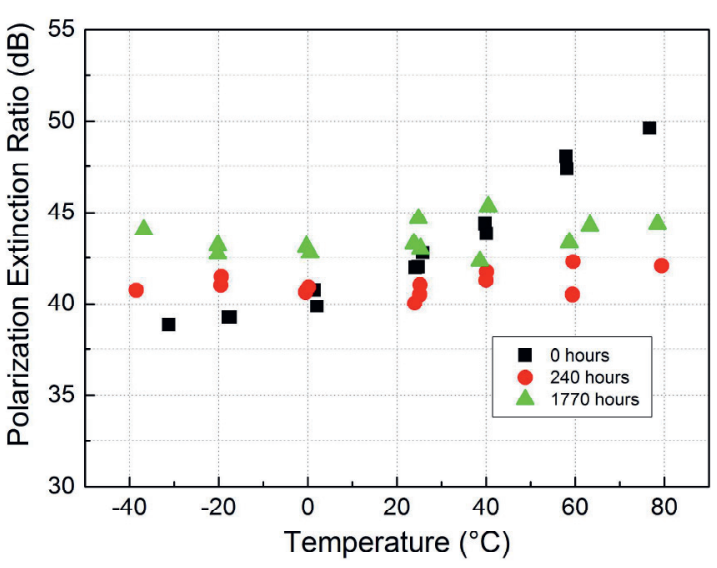

Fig. 4: Polarization extinction ratio of the modulator at a waveguide-to-fiber junction vs temperature after different ageing times.

$\sigma= \pm 200 \mathrm{pm}$, which is represented by the error bars. The SLEDs did not exhibit a significant wavelength shift after 7000 hours of testing time. The corresponding scale factor shift resulting from the variation of the wavelength was well below $\pm 0.1 \%$. In addition, the optical power did not show any obvious degradation over the test period.

Cross-coupling between the orthogonal polarization modes in the sensor's optical circuit can lead to a variation of the sensor scale factor. In particular, in the integrated-optic modulator, fiber stress within the fiber holders at the two end facets of the modulator chip can give rise to cross-coupling. Typically, such stress varies with temperature and time. Therefore, long-term stable operation calls for devices with high polarization extinction (PER) ratio (i.e. small cross-coupling) over large temperature range (ideally from -40 to $75^{\circ} \mathrm{C}$ ).

In addition, the sealing of the modulator package represents another reliability-critical aspect as it should provide a firm protection against moisture ingress. Humidity in the component package can degrade adhesives, which can result in an increase of the insertion loss due loss of optical alignment, and in severe cases even lead to total failure. At the same time, care must be taken that the fiber feedthroughs and adhesives do not produce any excessive, temperature-dependent mechanical stress on the optical fiber leads.

The modulators were characterized with regard to their insertion loss and, by using white light interferometry, their spatially resolved polarization extinction ratio (i.e. polarization cross-coupling along the optical path). Here, two orthogonal linearly polarized light waves with variable optical delay were launched in the modulator input fiber and brought to interference at an analyzer after the output fiber. The amplitude of the interference signal as a function of the delay represents the spatially resolved magnitude of the cross-coupling along the optical path.

As an example, Fig. 4 depicts the PER of a fiberto-modulator waveguide junction as a function of temperature after different ageing times. No remarkable degradation was encountered after 1770 hours of ageing. The polarization extinction ratio remained well above critical limits. In addition, the modulator's insertion loss and its temperature dependence remained stable over the entire testing time. Similar results were obtained for the other tested samples from the same supplier. It should be noted however, that failures can occur if modulators are not adequately sealed.

\section{Summary}

Damp heat tests of fiber-optic current sensor components were performed that are used in harsh environments. The performance of critical components, in particular superluminescent LEDs (SLEDs) and birefringence modulators, was periodically examined over extended periods of time. Appropriately designed and packaged components show stable performance which provide reliable sensor operation in the field.

\section{References}

[1] G. Frosio and R. Dändliker, Applied Optics, 33, 6111-6122 (1994).

[2] J. Blake, P. Tantaswadi, R.T. de Carvalho, IEEE Transactions on Power Delivery, 11, 116-121 (1996).

[3] K. Kurosawa, Optical Review 4, A38-A44 (1997).

[4] K. Bohnert, P. Gabus, J. Nehring, H. Brändle, Journal of Lightwave Technology, 20, 267-276 (2002).

[5] S. Mohr, T. Bosselmann, IEEE Sensors J. 3, 87-94 (2003).

[6] F. Rahmatian, G. Polovick, B. Hughes, V. Aresteanu, Cigre (Paris), Session 2004, paper A3-111.

[7] K. Bohnert, P. Gabus, J. Kostovic, and H. Brandle, Opt. Lasers Eng. 43, 511-526 (2005).

[8] K. Bohnert, P. Gabus, J. Nehring, H. Brändle, and M. G. Brunzel, J. Lightwave Technol. 25, 3602-3609 (2007).

[9] J. Wang, Z. Guo, G. Zhang, Proceedings APPEEC 2010 (Asia-Pacific Power \& Energy Engineering Conference 2010), Chengdu, China, 28-31 March 2010, 1178-1179 (2010). 
[10] K. Bohnert, A. Frank, T. Roininen, B. Gülenaltin, P. Gabus, A. Vujanic, IEEMA TECH-IT 2014 Technical digest, I-1-I-7 (2014).

[11] K. Kurosawa, Photon. Sens. 4, 12-20 (2014).

[12] H. Lefevre, "The fiber-optic gyroscope", Artech House, Boston (1993).

[13] M. Lenner, L. Yang, A. Frank, K. Bohnert, OSA Optical Sensors conference, paper ID 107778 (2014).

[14] M. Lenner, L. Yang, A. Frank, K. Bohnert, Proc. Eng. 168, 1735-1738 (2016).

[15] IEC 61300-2-19 standard, Part 2-19, 3rd edition (2012).

[16] A. H. Rose, S. M. Etzel, C. M. Wang, Journal of Lightwave Technology, 15, 803-807 (1997).

[17] M. Fukuda, Reliability and degradation of semiconductor lasers and LEDs, Artech House (1991). 